\title{
Parametric Geometric Model and Shape Optimization of Airfoils of A Biomimetic Manta Ray Underwater Vehicle
}

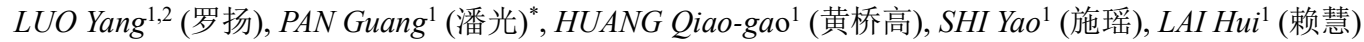 \\ (1. Key Laboratory for Unmanned Underwater Vehicle, Northwestern Polytechnical University, Xi'an 710072, China; 2. Department \\ of Naval Architecture, Ocean and Marine Engineering, University of Strathclyde, Glasgow G4 0LZ, Scotland, UK)
}

\begin{abstract}
As a new kind of autonomous underwater vehicle, bionic submersible has many merits such as high efficiency and low costs. To simulate the shapes of marine animals and apply them to the design of artificial underwater vehicle is a good way to obtain such advantages. In this paper, an optimization system of airfoils is proposed by the improved Class function /Shape function Transformation (CST) parameterization method and genetic algorithm (GA). The appearance of a manta-ray-inspired underwater vehicle is rebuilt using the optimal sectional airfoils obtained by the proposed optimization system. Computational simulations are carried out to investigate the hydrodynamic performance of the submersible using the commercial Computational Fluid Dynamics (CFD) code Fluent. The results demonstrate that the maximum thickness of the vehicle increases by $9 \%$, which means the loading capacity is increased. Moreover, the underwater vehicle shows better hydrodynamic performance, and the lift-drag ratio of initial design is increased by more than $10 \%$ using the presented optimization system of airfoils.
\end{abstract}

Key word: biomimetic underwater vehicle, CST parameterization method, shape optimization, computational fluid dynamics (CFD)

\section{CLC number: U $662 \quad$ Document code: A}

\section{Introduction}

In recent years, the underwater vehicles in rapid development have made great achievements in marine exploration ${ }^{[1]}$. The configurations of most of the existing underwater vehicles have revolving body, propeller and control surfaces. Even though they are easy to control, they fail to achieve excellent propulsion performance like most marine organisms. Natural fishes have many features suitable for the survival of underwater environment, and they can achieve extraordinary propulsion efficiencies, acceleration and maneuverability with low noise ${ }^{[2]}$. Therefore, it is of great significance to study bio-inspired shape to develop underwater vehicle with high

Received date: 2017-09-12.

*E-mail: panguang@nwpu.edu.cn

Foundation item: National Key Research and Development Plan of China (No. 2016YFC0301300) 
performance ${ }^{[3]}$.

Among millions of marine animals, manta ray is an atypical marine creature that moves forward by flapping its medium fins and/or paired fins (MPF) and gliding ${ }^{[4]}$. Plenty of research has been conducted on the development of manta-ray-inspired submersible so far ${ }^{[5-8]}$. In 2006 , Brower ${ }^{[9]}$ used several sequentially posed pictures of manta ray to produce the quantified dimensions of manta ray body and developed a manta ray based underwater vehicle. After few years, Wilfried ${ }^{[10]}$ illustrated an excellent design of ray-like fish robot. Driven by fluidic muscle, Aqua Ray can perform elegant swimming and gliding motions in water. According to biologic results ${ }^{[1]}$ and video analysis of free-swimming manta ray in marine museum, Cai et al. ${ }^{[12]}$ used NACA0020 to NACA0010 airfoils as cross sections to design a soft body underwater vehicle called Robo-ray II. More recently, Li et al. ${ }^{[13]}$ obtained the geometric abstraction of a manta ray from the dorsal and ventral views and hereby, they completed the design and modeling of a ray-like robotic fish.

However, current research on manta-ray-inspired underwater vehicle mainly focuses on propulsive efficiency. How to extract the body features from manta ray for modeling and simulation is seldom discussed, but it is indeed necessary to imitate the shape and propulsion better. As a matter of fact, the streamline shape of the manta ray contributes to the reduction of water resistance, while flattened and large body increases the swimming stability. And few manta ray based studies pay attention to gliding propulsion. Nevertheless, observations of manta rays indicate that manta rays adopt a combination of flapping fins and gliding propulsion. Even though gliding locomotion fails to achieve a speed as fast as flapping fins, it can save more energy, which makes the underwater vehicle more endurable in underwater cruising, and thus improve efficiency ${ }^{[14]}$. On the other hand, the research results show that the profile shape of the submersible plays an important role in affecting the gliding performance $\mathrm{e}^{[3 \text {, }}$ ${ }^{15]}$. An appropriate design of the geometrical configuration can not only reduce the resistance but also provide high lift for the underwater vehicle.

As a result, based on the previous research on manta ray, this paper focuses on shape design of a ray-inspired underwater vehicle. Here the gliding performance is the main consideration to simulate the propulsion of the manta ray. To achieve the gliding propulsion of the underwater vehicle with large lift-drag ratio, a manta ray based shape design is expected through the optimization of its cross section airfoils. The classic shape function / class function transformation (CST) parameterization method is improved to express the airfoils and Genetic Algorithm (GA) is introduced to solve the optimization. Better hydrodynamic performance of airfoils is obtained after the optimization. 
And the ray-like underwater vehicle can glide underwater with larger lift-drag ratios using the optimal airfoils.

\section{1. the Optimization System of Airfoil}

\subsection{CST Parameterization Method}

The improved CST method ${ }^{[16,17]}$ is employed to construct the parameterized model of the airfoil. The airfoil expression generated can be written as

$$
\begin{gathered}
y_{\mathrm{u}}(x)=y_{\mathrm{u}, 0}(x)+x^{N 1} \cdot(1-x)^{N 2} \cdot \sum_{i=0}^{N} A_{\mathrm{u}, i} S_{i}(x), \\
y_{1}(x)=y_{1,0}(x)+x^{N 1} \cdot(1-x)^{N 2} \cdot \sum_{i=0}^{N} A_{1, i} S_{i}(x),
\end{gathered}
$$

where the length of the airfoil is unit length, $x$ is the abscissa, $y_{0}$ is the coordinate of base airfoil, $y$ represents the coordinate of new airfoil, the subscripts $u$ and 1 respectively represents the upper and lower surface of the airfoil, $A_{\mathrm{u}, i}$ and $A_{1, i}$ are undetermined coefficients, while $S_{i}(x)$ implies Bernstein polynomial. For a round nose airfoil considered here, $N 1=0.5$ and $N 2=1.0$

It is reasonable to choose an appropriate order during using the improved CST method. A very low order will lead to low fitting accuracy, but an exorbitant one is also unfavorable. The design practice shows that the satisfactory accuracy can be obtained by using the $4^{\text {th }}$ order Bernstein polynomial, so we choose $5^{\text {th }}$ order, i.e. $N=5$. The leading edge radii of the upper and lower surface of the airfoil should be consistent, so the improved CST parameterization contains 11 parametric variables:

$$
\left\{\begin{array}{l}
\boldsymbol{X}_{\mathrm{u}}=\left\{A_{\mathrm{u}, 0}, A_{\mathrm{u}, 1}, A_{\mathrm{u}, 2}, A_{\mathrm{u}, 3}, A_{\mathrm{u}, 4}, A_{\mathrm{u}, 5}\right\} \\
\boldsymbol{X}_{1}=\left\{A_{1,1}, A_{1,2}, A_{1,3}, A_{1,4}, A_{1,5}\right\}
\end{array} .\right.
$$

\subsection{GA}

The $\mathrm{GA}^{[18]}$ is used in the optimization system of airfoil for its high efficiency and convenience. GA mimics biological evolution law of natural selection. It can decode data structures of chromosomes in some specific problems and apply restructuring operation on them to conserve key information. The general procedure of GA can be depicted, as shown in Fig. 1.

\subsection{Establishment of the Optimization System of Airfoil}

In this paper, the improved CST parameterization method, XFOIL solver and GA are integrated to form the optimization system of airfoil. The objective function for the design of the airfoils of ray-inspired underwater 
vehicle can be expressed as

$$
\operatorname{Obj}=\operatorname{Minimize}\left(-C_{L} / C_{D}\right)
$$

where $C_{L}$ is the lift coefficient, and $C_{D}$ is the drag coefficient. Two groups of parametric variables of the airfoils whose thicknesses approximate to that of the base airfoils are used as constraint conditions. For example, if the base airfoil is NACA0012 and the thickness of new airfoil is expected to range from NACA0014 to NACA0016. Then they are fitted using CST parameterization method and two groups of parametric variables corresponding to different thicknesses are obtained. The smaller one is selected as lower limit while the larger as upper limit. The design variables are expressed as

$$
\boldsymbol{X}=\left\{x_{0}, x_{1}, x_{2}, x_{3}, x_{4}, x_{5}, x_{6}, x_{7}, x_{8}, x_{9}, x_{10}\right\} .
$$

It can be seen from the above description that the optimization of airfoil in this paper is a typical single-objective nonlinear optimization issue. The flowchart of the proposed optimization system is illustrated in Fig. 2.

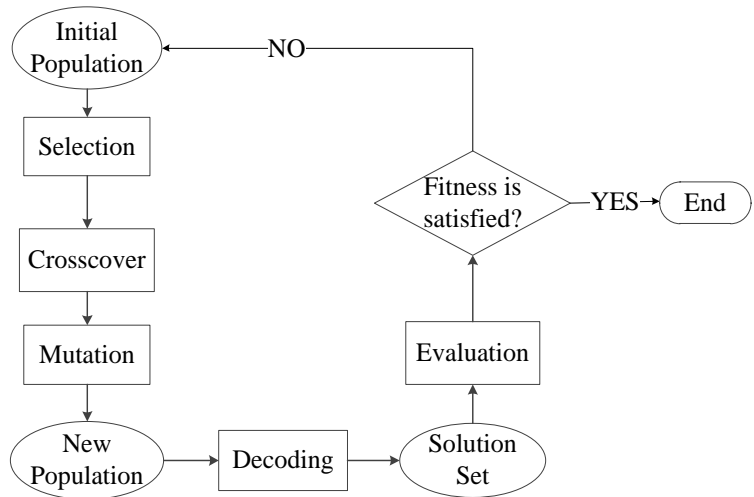

Fig. 1 The general procedure of GA

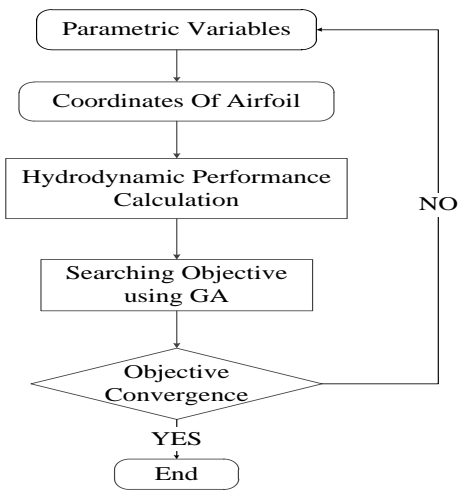

Fig. 2 The optimization flowchart

\section{Application of the Optimization System}

\subsection{Selection of Base Airfoil and Its Station}

Figure 3 shows a manta ray in swimming and Fig. 4 indicates the distribution of sections. According to different shape characteristics and body functions, a manta ray is considered to consist of two parts: body and pectoral fins. It is divided by three sections, as shown in Fig. 4. Li G et al. ${ }^{[13]}$ and Yang $\mathrm{S}^{[6]}$ extracted the geometric abstraction of a manta ray, and their findings both suggest that the maximum thickness of the body is about 2/9 longitudinal body length. It has similar thickness characteristics with NACA0022. So NACA0022 is used as base airfoil in Section 1. And to achieve smooth transition from body and fin tips, NACA0016 is elected in Section 2 and NACA0008 in Section 3. 


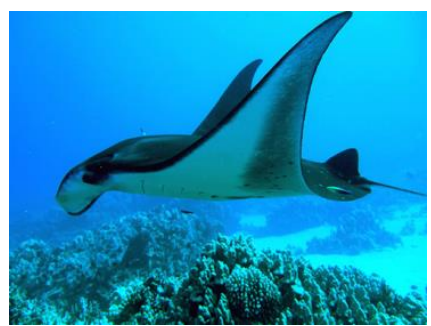

Fig. 3 A manta ray in swimming

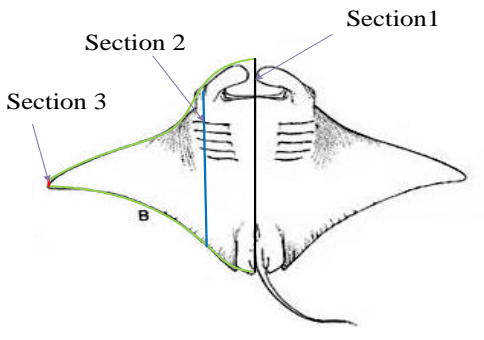

Fig. 4 Selection of different Sections

\subsection{Optimization Design of the Airfoil}

NACA0016 in Section 2 is taken as an example to illuminate the optimization. The population and the number of elites are 200 and 10 respectively in GA. The design state is $R e=10^{6}, M a=0, \alpha=0 \sim 10^{\circ}$, where $M a$ is Mach number, Re is Reynolds number and $\alpha$ is the angle of attack. And design target is $\left(C_{L} / C_{D}\right)_{\max }$. The range of parametric variables and optimal results are shown in Table 1 and the optimal airfoil and NACA0016 are depicted in Fig. 5, where $c$ denotes the chord length of the airfoil. In addition, for investigating the optimization effect, $C_{L}$, $C_{D}$ and $C_{L} / C_{D}$ of the airfoils are compared in Fig. 6. It can be seen that the airfoil with a clear streamline shape is more similar to the side profile of a manta ray after optimization. As shown in Fig. 6, the optimized airfoil obtains better hydrodynamic performance whose lift-drag ratio improves greatly in the calculation range of angle of attack.

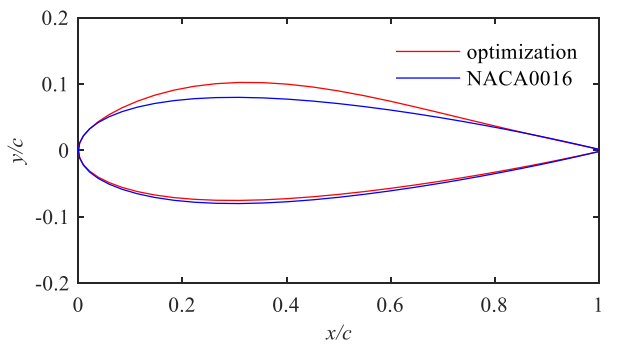

Fig. 5 The shape of airfoils

Table 1 The design range of parametric variables and optimized results

\begin{tabular}{cccc}
\hline Parametric Variable & Lower Bound & Upper Bound & Optimization Result \\
\hline$x_{0}$ & -0.01419 & 0.08519 & -0.00935 \\
$x_{1}$ & -0.01335 & 0.08008 & 0.08007 \\
$x_{2}$ & -0.01196 & 0.07183 & 0.07178 \\
$x_{3}$ & -0.01388 & 0.08320 & 0.06667 \\
$x_{4}$ & -0.00918 & 0.05524 & -0.00761 \\
$x_{5}$ & -0.01496 & 0.08970 & -0.01216 \\
$x_{6}$ & -0.08008 & 0.01335 & 0.01335 \\
$x_{7}$ & -0.07183 & 0.01196 & 0.01194 \\
$x_{8}$ & -0.05524 & 0.00918 & 0.01387 \\
$x_{9}$ & -0.08970 & 0.01496 & 0.00918 \\
$x_{10}$ & -0.01419 & 0.08519 & 0.01480 \\
\hline
\end{tabular}


To explain the improvement of fluid dynamic performance, the polar curves and flow transition positions of the upper surface of the two airfoils are compared in Fig. 7 and Fig. 8, where Topxtr denotes the relative position of the transition point at the upper airfoil surface with respect to its chord length. It can be found that in most cases, the lift coefficient of the airfoil after optimization is much larger when their resistance coefficients are thesame. And the laminar-turbulent transition is successfully delayed to reduce drag when the angle of attack is beyond 1 degree.

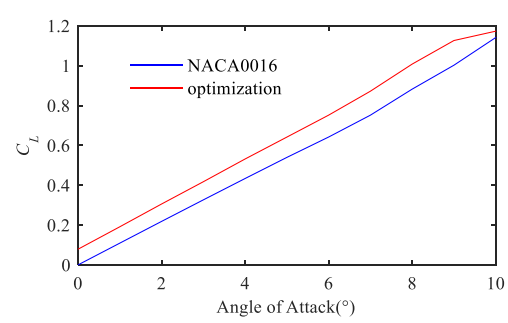

(a) $C_{L}$

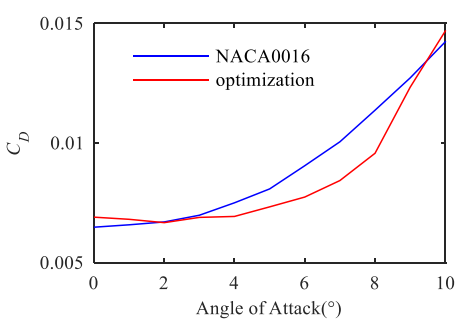

(b) $C_{D}$

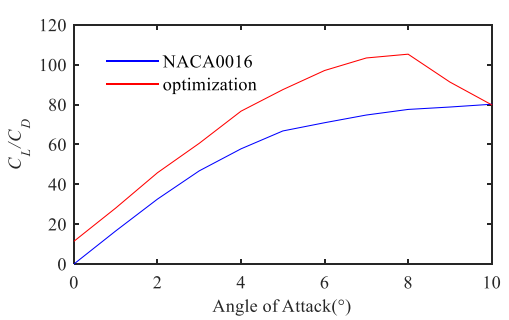

(c) $C_{L} / C_{D}$

Fig. 6 Fluid dynamic coefficients

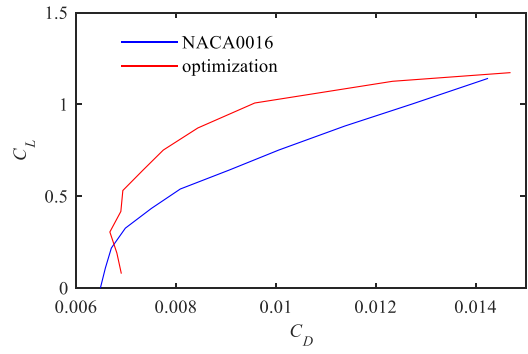

Fig. 7 The polar curves

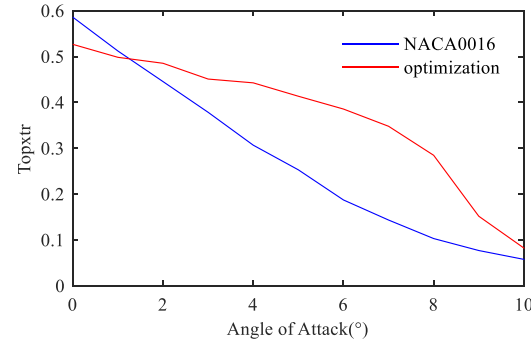

Fig. 8 The laminar-turbulent transition

\section{3D Shape Design of the Manta Ray Inspired Submersible}

In this paper, Fig. 9 is taken as blueprint. Pectoral fins and body are taken into consideration only, while the head fins, eyelid and eye gills of the manta ray are neglected. And the dorsal profile of the manta ray is divided into four segments which are fitted with four quadratic functions as formula (6). The fitted outline of the manta ray is shown in Fig. 10.

The optimized airfoils in three stations are used as base contour to be swept along with the fitted curve, and the smooth transition from body to fins is achieved. The 3D configuration of the manta ray based underwater vehicle is shown in Fig. 11. Its dimension is: the length $L=1000 \mathrm{~mm}$, the wing span $C=1968 \mathrm{~mm}$ and the maximum thickness $D=240 \mathrm{~mm}$, so the span over length ratio is about 1.9. The 3D shapes of the submersibles with the same dorsal profile using base and optimized airfoils are compared in Fig. 12. 


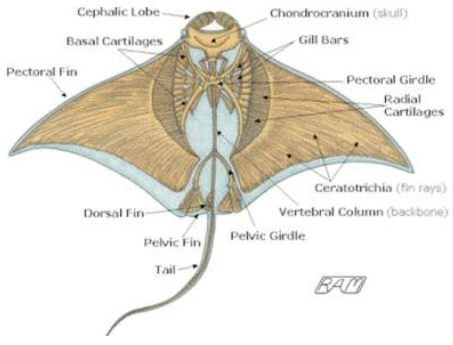

Fig. 9 Skeleton structure diagram of a manta ray

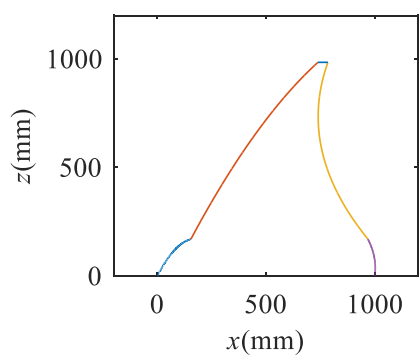

Fig. 10 The fitted outline

$$
\left\{\begin{array}{l}
z(x)=-0.005477 \cdot x^{2}+1.942 \cdot x+3.383 \mathrm{e}-14(0 \leq x<153.8) \\
z(x)=-0.0008236 \cdot x^{2}+2.129 \cdot x-138.8(153.8 \leq x<738.24) \\
x(z)=0.0007184 \cdot z^{2}-1.055 \cdot z+1127(763.7584 \leq x<968.94) \\
x(z)=-0.001924 \cdot z^{2}+0.1419 \cdot z+1000(968.94 \leq x<1000)
\end{array} .\right.
$$

Seen from Fig. 11, the side contour is more similar to that of a manta ray when the airfoils after optimization are used, thus the underwater vehicle has a more similar overall appearance as well. Moreover, the maximum thickness increases from $220 \mathrm{~mm}$ to $240 \mathrm{~mm}$ which enlarges the vehicle's capacity. Another advantage found in Fig. 12 is that the thickness of the distal pectoral fin of the vehicle also increases, which enhances the ability to resist damage of structure while flapping fins.

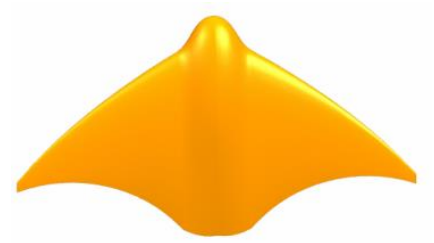

(a) Top view

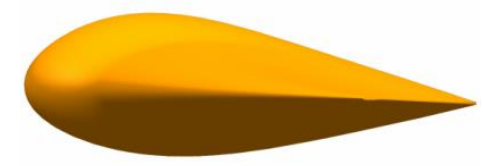

(b) Side view

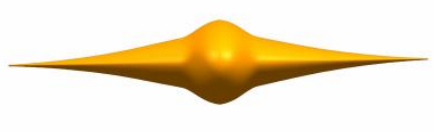

(c) Front view

Fig. $113 \mathrm{D}$ configuration of the underwater vehicle using optimized airfoils

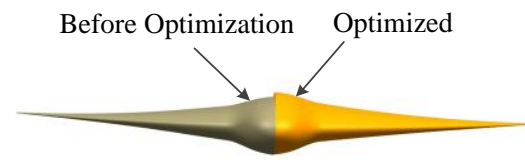

(a) Front view

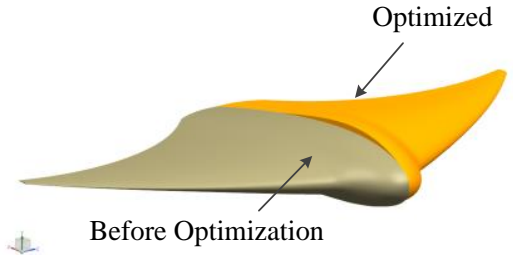

(b) Zoom in the symmetrical surface

Fig. 12 Comparison of vehicles before and after optimization

\section{CFD Simulations}

\subsection{Governing Equations}

The hydrodynamic numerical simulations of the biomimetic manta ray underwater vehicle presented in this paper are carried out using the commercial code Fluent, based on finite volume method. The governing equations are the Navier-Stokes equations for incompressible viscous flow including mass and momentum conservations forms, 
written as:

$$
\begin{gathered}
\nabla \cdot v=0 \\
\frac{\partial v}{\partial t}+(v \cdot \nabla) v=-\frac{1}{\rho} \nabla p+\frac{\mu}{\rho} \nabla^{2} v
\end{gathered}
$$

where $\rho$ is the density of fluid, $v$ represents velocity vector, $p$ is the pressure, and $\mu$ refers to the stress tensor.

\subsection{Numerical Method Validation}

In order to verify the accuracy of numerical calculation method, the hydrodynamic performance of a 3D plate hydrofoil is calculated. The experimental data ${ }^{[19]}$ and the numerical results are compared in Fig. 13. The simulation is conducted while $R e=1.35 \times 10^{5}$ in water.

As shown in Fig. 13, the simulation lift coefficients are little smaller than the experimental data while the predicted drag coefficients are bigger than the experiment counterparts. In general, they have a good agreement. So the hydrodynamic performance of the underwater vehicle can be investigated using the CFD simulation.

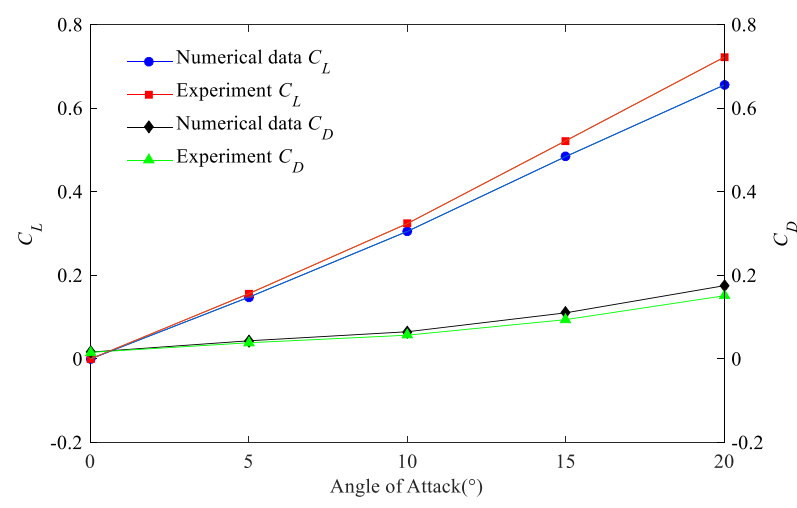

Fig. 13 Comparison of CFD results with experiment results

\subsection{Analysis of Hydrodynamic Performance of the Underwater Vehicle}

The steady dive of the underwater vehicle at a velocity of $1 \mathrm{~m} / \mathrm{s}$ is calculated. Under the condition, the attack angles range from 0 degree to 10 degree with an interval of 1 degree. The grid is divided into structured grids, and the amount is about $1.5 \times 10^{6}$ to $1.8 \times 10^{6}$. The shear-stress transport (SST) $k-\omega$ turbulence model for incompressible flow is selected. The hydrodynamic coefficients obtained are shown in Fig. 14, and the pressure distribution of the surface of the vehicles is compared in Fig. 15. 


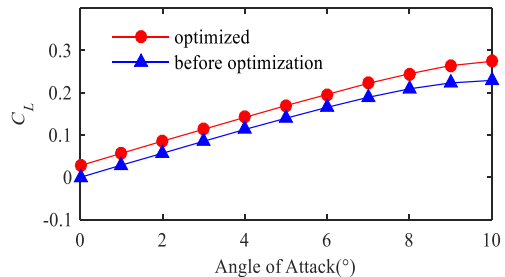

(a) $C_{L}$

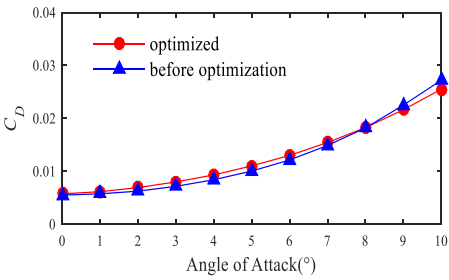

(b) $C_{D}$

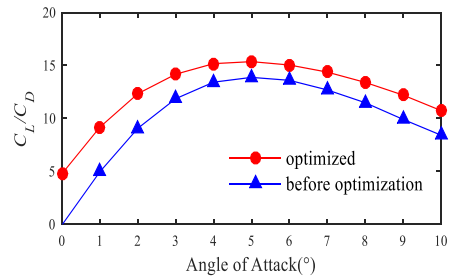

(c) $C_{L} / C_{D}$

Fig. 14 The hydrodynamic coefficients in the angle of attack $0 \sim 10^{\circ}$

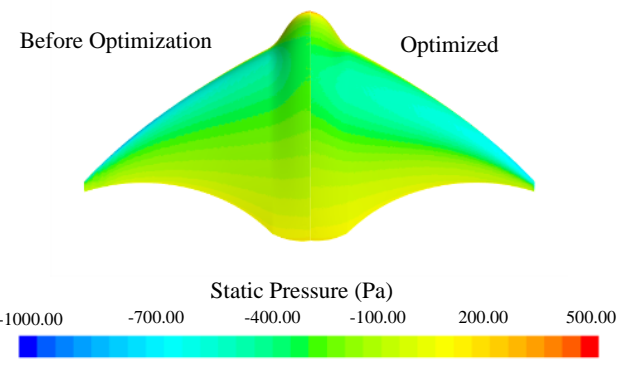

(a) Upper surface

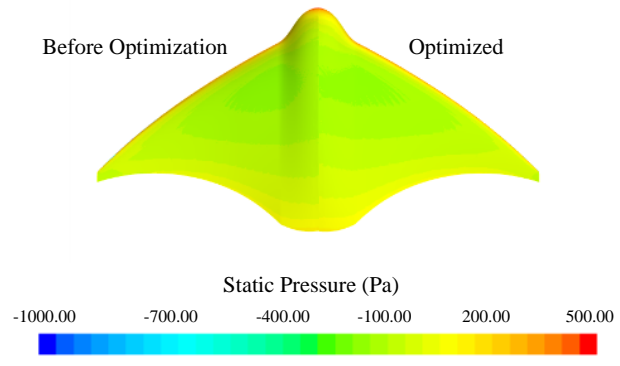

(b) Lower surface

Fig. 15 The pressure distribution of the surface of the vehicles in the angle of attack $5^{\circ}$

Within the range of the angle of attack $0 \sim 10$ degree, the lift coefficient improves while the drag coefficient is generally reduced. And lift-drag ratios increase by more than $10 \%$, even by $84.67 \%$ at 1 degree. Their maximum ratios of lift to drag reach 15.36 and 13.87 respectively at the gliding angle of $5^{\circ}$. This can demonstrate that the hydrodynamic performance of the submersible improves using the optimal airfoils obtained by the proposed optimization system.

As shown in Fig. 15, the low pressure covers more upper surface of the vehicle after the optimization, which indicates that the accelerating pressure gradient influences more widely in the boundary layer. So it contributes to the delay of the transition from lamina to turbulence and then the reduction of resistance. Overall, the pressure difference between the upper and lower surface is larger and the lift to drag ration also improves after the optimization.

\section{Conclusion}

In this paper, the hydrodynamic structure shape of a biomimetic underwater vehicle inspired by the appearance of manta rays is built up. In order to imitate the gliding propulsion of the manta ray with high lift-drag ratio, an optimization system for airfoils with 11 design variables is established by CST parameterization method. The GA is introduced to solve the hydrodynamic design optimization of airfoils. After the 3D shape design of underwater vehicle is completed, a Navier-Stokes code is adopted to evaluate the hydrodynamic performance of the vehicle 
during gliding underwater. By summarizing the results of this study, we conclude as follows.

(1) The airfoils are more similar to the cross profile of a manta ray and better hydrodynamic performance is obtained after the optimization.

(2) The payload is increased because of the increase of the maximum thickness of the vehicle. And the ability to resist damage of structure with flapping fins is also enhanced for the thicker distal pectoral fin.

(3) The hydrodynamic performance of the vehicle during gliding underwater improved obviously (rise by more than $10 \%$ ) after the optimization of the airfoils.

\section{References}

[1] YOERGER D R, JAKUBA M, BRADLEY A M, et al. Techniques for deep sea near bottom survey using an autonomous underwater vehicle [J]. The International Journal of Robotics Research, 2007, 26(1): 41-54.

[2] TRIANTAFYLLOU M S, TRIANTAFYLLOU G S. An efficient swimming machine [J]. Scientific american, 1995, 272(3): 64-70.

[3] WANG Z, Yu J, ZHANG A. Hydrodynamic performance analysis of a biomimetic manta ray underwater glider[C]// Proceedings of the 2016 IEEE International Conference on Robotics and Biomimetics (Robio). Qingdao, China: IEEE, 2016: 1631-1636.

[4] ROSENBERGER L J. Pectoral fin locomotion in batoid fishes: undulation versus oscillation [J]. Journal of Experimental Biology, 2001, 204(2): 379-94.

[5] SUZUMORI K, ENDO S, KANDA T, KATO N, SUZUKI H. A bending pneumatic rubber actuator realizing soft-bodied manta swimming robot[C]// Proceedings of the 2007 IEEE International Conference on Robotics and Automation. Roma, Italy: IEEE, 2007: 4975-4980.

[6] YANG S-B, QIU J, HAN X-Y. Kinematics modeling and experiments of pectoral oscillation propulsion robotic fish [J]. Journal of Bionic engineering, 2009, 6(2): 174-9.

[7] CAI Y, BI S, ZHENG L. Design optimization of a bionic fish with multi-joint fin rays [J]. Advanced Robotics, 2012, 26(1-2): 177-96.

[8] CHEN Z, UM T I, BART-SMITH H. Bio-inspired robotic manta ray powered by ionic polymer-metal composite artificial muscles [J]. International Journal of Smart and Nano Materials, 2012, 3(4): 296-308.

[9] BROWER T P L. Design of a manta ray inspired underwater propulsive mechanism for long range, Low Power Operation [D]. Boston, USA: Tufts University, 2006.

[10] WILFRIED S. Water-hydraulic manta ray with flapping-wing drive [EB/OL].(2015-09-20). https://www.festo.com/rep/en_corp/assets/pdf/Aqua_ray_en.pdf..

[11] CLARK R P, SMITS A J. Thrust production and wake structure of a batoid-inspired oscillating fin [J]. Journal of fluid mechanics, 2006, 562(415-29.

[12] CAI Y, BI S, ZHENG L. Design and experiments of a robotic fish imitating cow-nosed ray [J]. Journal of Bionic Engineering, 2010, 7(2): 120-6.

[13] LI G, DENG Y, OSEN O L, et al. A bio-inspired swimming robot for marine aquaculture applications: From concept-design to simulation[C]// Proceedings of the OCEANS 2016-Shanghai. Shanghai, China: IEEE, 2016: 1-7.

[14] ZHOU C, LOW K-H. Better endurance and load capacity: an improved design of manta ray robot (RoMan-II) [J]. Journal of Bionic Engineering, 2010, 7(S137-S44.

[15] SUN C, SONG B, WANG P. Parametric geometric model and shape optimization of an underwater glider with blended-wing-body [J]. International Journal of Naval Architecture and Ocean Engineering, 2015, 7(6): 995-1006.

[16] KULFAN B M. Universal parametric geometry representation method [J]. Journal of Aircraft, 2008, 45(1): 142-58.

[17] BU Y, SONG W, HAN Z, et al. Aerodynamic optimization design of airfoil based on CST parameterization method [J]. Journal of Northwestern Polytechnical University, 2013, 31(5): 829-36 (in Chinese).

[18] GOLDBERG D E, HOLLAND J H. Genetic algorithms and machine learning [J]. Machine learning, 1988, 3(2): 95-9.

[19] CHENG X. Theoretical and experimental study on the leading-edge tubercle of three-dimensional airfoil [D]. Harbin, China: Harbin Engineering University, 2013 (in Chinese). 